\title{
Acoustic dispensing-mass spectrometry: the next high throughput bioanalytical platform for early drug discovery
}

\author{
Hui Zhang ${ }^{*} 1$ \\ ${ }^{1}$ Discovery Sciences, Pfizer Inc., Groton, CT, 06340, USA \\ * Author for correspondence: Tel.: +1 860686 0449; hui.zhang3@pfizer.com
'Gacoustic dispensing-MS could be an ideal tool to sample and detect small amount of products from thousands or more chemical reactions conducted in parallel."

First draft submitted: 2 May 2017; Accepted for publication: 20 July 2017; Published online: 2 November 2017

Keywords: acoustic dispensing-MS • ADME • HTS • label-free • nanoliter sampling

Early drug discovery is an extremely daunting task, from identifying the initial hit(s), to selecting the right lead series with balanced efficacy, safety and ADME properties. The process of early drug discovery generally starts with a large library (which contains anywhere from thousands to millions of compounds) and ends up with only a handful of lead molecules that go on to enter the later discovery/development stage. Throughput is mandatory considering the vast number of new chemical entities and different type of assays that need to be screened through for timely decision-making.

\section{Seeking for the high throughput with MS}

MS is readily available to provide selective and sensitive detection/characterization of a wide range of analytes, including small pharmaceutical drug-like molecules, lipids, peptides, proteins as well as many more. Different types of MS have found their ways in various bioassays performing high throughput screening within early drug discovery stages. For example, both matrix-assisted laser desorption/ionization [1] and laser diode thermal desorption-MS [2] have successfully demonstrated utility for certain in vitro ADME screening, however such application are limited because neither approach can provide universal detection (limited by the nature of ionization). ESI based LC/MS is a more powerful approach as it covers a vast number of analytes; it is not surprising that LC/MS has become the dominant platform and workhorse for early discovery (e.g., ADME, PK etc.) with different types of instrumentation on the market (e.g., RapidFire [Agilent Technologies, CA, USA] [3], Apricot Designs Dual Arm [ADDA; Apricot Designs, CA, USA] [4], Parker [Parker, OH, USA] [5] etc.) already. Combined with fast LC separation, such systems can reliably deliver 10-20 s/sample speed (or 1-2 h/384-well plate). Considering the physical limit of liquid handling and delivery by syringe injection, it is not likely to reach sub s/sample (a few min/plate) speed, which most colorimetric based plate readers can readily provide. To the author this is the single biggest hurdle that MS-based platforms need to overcome before they find a broader utility in the early drug discovery screening space, such as pharmacological high throughput screening (HTS). In particular, a different sampling technique would be needed for MS analysis other than conventional syringe/needle approach.

\section{Getting faster with acoustic technology}

Acoustic dispensing is a very attractive option by which precisely controlled nanoliter liquid droplets can be generated and delivered via fine-tuned ultrasonic acoustic energy. Acoustic technology based instruments have been widely adopted within pharmaceutical companies (mostly for compound logistics) as it provides fast and reliable sample delivery in microtiter plate format. When combined with MS, acoustic dispensing can also solve the sampling throughput bottleneck aforementioned, the sampling speed would be mostly limited by the movement of mechanical stage that holds the sample plate, while the sample droplets can be acoustically dispensed in a fraction 
of a second. As demonstrated by AstraZeneca/Labcyte/Waters groups recently [6], acoustic dispensing sampling device was successfully integrated with a conventional mass spectrometer. By eliminating the injection needle and LC separation, liquid samples can be introduced into the mass spectrometer for detection at very fast speed (up to $3 \mathrm{~Hz}$ overall sampling speed). The very first screening campaign has successfully finished with positive hits to a reductase target being identified from a large compound library $(>300 \mathrm{~K})[7]$. With the very first demonstration of using an MS end point as a real large scale HTS campaign, there is less doubt that the MS based screening tool has become a reality and more and more Pharma are embracing this new revolution.

Before the full celebration and welcoming of the era of using acoustic-MS for all HTS, further optimization and developments are required to make it more widely applicable. For one, the detection sensitivity has to be improved significantly for the majority of the HTS. The current substrate concentration used in AstraZeneca's case [7] (micromolar range) was orders higher than routine HTS (many of which are in nanomolar range). For certain cases such high concentration requirement could be cost prohibitive, for a large scale screening campaign. Second, the reproducibility should be further improved, for example the current raw data being presented exhibited great variability for the substrate signals from different wells even though they were dosed at the same concentration. Internal standard could help to normalize (as current practice), however; clearly there are great spaces to improve, especially when compared with the performance benchmark of standard LC-MS techniques. For the current setup used in the AstraZeneca/Labcyte/Waters studies, nanoliter droplets were broke down to even smaller mists (picoliter sizes) with addition of special detergent, a very critical component judiciously selected to facilitate the ionization and improve transferring efficiency of the mists. The detergent could also bring in the two major limitations inevitably: the detergent may interfere with MS detection, this in combination with the low ionization efficiency could be the explanation of the poor detection sensitivity; the smaller mists are generally harder to control compared with nanoliter droplets, which could be the root cause of the variability.

\section{Conclusion \& future perspective}

Further efforts are granted for a better interface between acoustic droplets generation and the MS orifice for sample introduction in order to enable more efficient transferring of the droplets and ionization. Also skipping the detergent would be ideal to facilitate the higher detection sensitivity and simplify the assay development, after all one big advantage to use MS detection is to enable direct and label-free detection with minimum assay optimization (such as skipping the substrate labeling steps as required for colorimetric methods). With that in place (under development in labs like ours already) more exciting development and applications using acoustic dispensing-MS predicated platform would emerge in early drug discovery especially in ADME and HTS applications. In addition, a clear trend has emerged for miniaturization (nanomole scale as demonstrated in recent Merck publication) [8] of medicinal chemistry synthesis in order to scout broader synthetic routes as well as to cover more chemical spaces in order to find better drug candidates. Therefore, acoustic dispensing-MS could be an ideal tool to sample and detect small amount of products from thousands or more chemical reactions conducted in parallel.

Financial \& competing interests disclosure

The author has no relevant affiliations or financial involvement with any organization or entity with a financial interest in or financial conflict with the subject matter or materials discussed in the manuscript. This includes employment, consultancies, honoraria, stock ownership or options, expert testimony, grants or patents received or pending, or royalties.

No writing assistance was utilized in the production of this manuscript.

\section{References}

1. Haslam C, Hellicar J, Dunn A et al. The evolution of MALDI-TOF mass spectrometry toward ultra-high-throughput screening: 1536-well format and beyond. J. Biomol. Screen. 21(2), 176-186 (2016).

2. Haarhoff Z, Wagner A, Picard P, Drexler DM, Zvyaga T, Shou W. Coupling laser diode thermal desorption with acoustic sample deposition to improve throughput of mass spectrometry-based screening. J. Biomol. Screen. 21(2), 165-175 (2016).

3. Leveridge M, Buxton R, Argyrou A et al. Demonstrating enhanced throughput of RapidFire mass spectrometry through multiplexing using the JmjD2d demethylase as a model system. J. Biomol. Screen. 19(2), 278-286 (2014).

4. Janiszewski J, Schneider R, Kapinos B et al. Development of a high-speed, multiplexed sample-delivery instrument for LC-MS/MS bioanalysis. Bioanalysis 4(9), 1039-1056 (2012).

5. Zelesky V, Schneider R, Janiszewski J, Zhang H. High Throughput LC/MS Methods Development for in vitro ADME Screening. Presented at: American Society of Mass Spectrometry. Denver, CO, USA, 5-9 June 2011. 
6. Sinclair I, Stearns R, Pringle $S$ et al. Novel acoustic loading of a mass spectrometer: toward next-generation high-throughput MS screening. J. Lab. Autom. 21(1), 19-26 (2016).

7. Wingfield J. High throughput acoustic mass spectrometry: development and delivery of a biochemical screen. SLAS (2017).

8. Buitrago Santanilla A, Regalado EL, Pereira T et al. Organic chemistry. Nanomole-scale high-throughput chemistry for the synthesis of complex molecules. Science 347(6217), 49-53 (2015). 
